\title{
LITERATURA Y CIENCIA EN LA COMPOSICIÓN MINIMALISTA: HACIA UNA TEORÍA DEL AZAR CONTROLADO
}

\section{LITERATURE AND SCIENCE IN MINIMALIST COMPOSITION: LOOKING FOR A THEORY OF CONTROLLED CHANCE.}

\author{
Marta Cureses \\ Universidad de Oviedo
}

\section{ABSTRACT}

Literature and science are both the fundamental pillars on which we try here to define a theory of controlled chance through musical minimalism and random composition. Literature — prose and poetry — and science - mathematics and music- are joined by another discipline taking part of the process: architecture, not in a generalist consideration, but from the very concrete approach proposed by Peter Eisenman's theory of architecture as a text.

Key words: literature theory, combinatorics, random music, minimalism, architecture.

\section{RESUMEN}

Literatura y ciencia son los dos pilares fundamentales sobre los que se trata aquí de definir una teoría del azar controlado en la composición minimalista y de signo aleatorio. Junto a la literatura — prosa y poesía- y ciencia - matemáticas y música- surge otra disciplina que 
forma parte del proceso: la arquitectura; no en una definición generalista, sino desde el planteamiento concreto que propone Peter Eisenman en su teoría de la arquitectura como texto.

Palabras clave: teoría literaria, combinatoria, aleatoriedad, minimalismo, arquitectura.

Fecha de recepción: 17 de junio de 2019.

Fecha de aceptación: 8 de agosto de 2019.

Cómo citar: Cureses, Marta (2019): «Literatura y ciencia en la composición minimalista: hacia una teoría del azar controlado», en Actio Nova: Revista de Teoría de la Literatura y Literatura Comparada, 3: 407-438.

DOI: https://doi.org/10.15366/actionova2019.3.018 


\section{INTRODUCCIÓN}

En el prólogo que Raymond Queneau redacta para una de las ediciones de sus Exercises de style, recuerda un concierto al que asistió junto a Michel Leiris en los años treinta en la sala Pleyel de París. Se interpretaba Die Kunst der Fuge de J. S. Bach. Al término del concierto ambos pensaron en hacer algo similar en el ámbito literario, prescindiendo de los elementos propios del contrapunto y la fuga para centrarse en un tema casi trivial, susceptible de variarse hasta el infinito

Antes de dedicarse a la composición, cuando Michael Nyman ejercía la crítica musical — hacia 1968 - descubrió la obra de los americanos Philip Glass, Steve Reich y Terry Riley, creaciones minimalistas entonces de carácter minoritario que se proyectarían después hacia el gran público europeo, más bien con un sentido divulgativo, sobre todo a través de la obra de Glass. Cuando Nyman comienza a colaborar con el director de cine Peter Greenaway (1977), su discurso adopta una narrativa compleja surgida de la teoría combinatoria: un procedimiento matemático, atractivo y aparentemente muy novedoso, inspirado en la escritura barroca de Henry Purcell.

En los planteamientos de Nyman y Queneau subyace un concepto de operación matemática de apariencia simple, un juego con los componentes fonéticos y literarios también plásticos - de libertad expresiva ajena a cualquier rigidez propia de un sistema. La introducción del azar como principio creativo en la composición —literaria, musical, arquitectónica - tiene una proyección posterior fundamental, especialmente en el ámbito francés, desde donde llega también a España. La aplicación de funciones estadísticas a la creación sonora en la era digital no cierra el círculo; más bien amplía el espectro de posibilidades involucrando al texto literario, poético, como parte del proceso.

Literatura y ciencia son aquí los dos pilares fundamentales sobre los que trataremos de exponer una teoría del azar controlado a través de las creaciones literarias —y musicalesde Raymond Queneau, Paul Braffort, Georges Perec, Michael Nyman y Josep M. Mestres Quadreny. En este proceso surge otra disciplina que forma parte del mismo proceso: la arquitectura; no en una definición generalista sino desde el planteamiento muy concreto que propone Peter Eisenman en su teoría de la arquitectura como texto.

Si nos atenemos a la teoría que Roland Barthes explica en Le bruissement de la langue (1984), la literatura presenta las características secundarias de una ciencia, o lo que es lo mismo, todas aquellas que no la definen. Y, al igual que la ciencia, posee su propia 
metodología y reglas de investigación. Pero si el rasgo común a ambas es el discurso, la manera en que una y otra asumen el lenguaje las separa: «Le discours scientifique croit être un code supérieur; l'écriture veut être un code total» (Barthes, 1984: 46). En cuanto al rumbo divergente de ambos discursos, la cita de Coleridge esclarece su objeto: «A poem is that species of composition which is opposed to works of science, by purposing, for its inmediate object, pleasure, not truth» ${ }^{1}$. Con el fin, pues, de enunciar un teoría del azar controlado a través de cuatro ámbitos de conocimiento distintos y de la interacción que se genera entre ellos de manera efectiva en una praxis multidisciplinar, tenemos aquí en cuenta diversas teorías propias de cada uno de ellos que nos permiten generar un corpus nuevo a partir de dos direcciones fundamentales: un componente de especulación específico para cada una de esas disciplinas o ámbitos y una serie verificada de proposiciones en el sentido más sencillo y directo, a la manera de Jonathan Culler (1997): una teoría que rebase la noción de mera hipótesis, que implique relaciones complejas de tipo sistemático entre diversos factores y, lo que es más importante para su formulación, que no se demuestre ni descarte con facilidad y dificulte la definición de sus límites. A estas señas de identidad se añaden otros cuatro rasgos que determinan su eficacia: la teoría es por definición interdisciplinar y trasciende su ámbito de origen, es analítica y especulativa, pone en cuestión conceptos asumidos, indaga en las categorías establecidas y se traduce en thinking about thinking, en reflexión.

Diversos aspectos de la teoría literaria se extienden hacia el contexto del azar — que tanta literatura ha generado-y se combinan necesariamente con aquellos que, procedentes sobre todo de la teoría de la probabilidad, encajan en este planteamiento. Dicho de otro modo, no es posible plantear esta teoría mediante un único método distintivo, pues, de hecho, como sucede en los métodos que intervienen en la crítica literaria, los que actúan en este proceso carecen de significación común y cada uno de ellos comparte mucho con otras disciplinas (Eagleton, 1983). Ello no impide que la interacción entre teoría literaria e historia de la literatura se produzca de idéntico modo entre el arte sonoro y su historia, con un efecto similar al que describe Hans Robert Jauss en su conocida lección inaugural de la Universidad de Constanza, «Literaturgeschichte als Provokation der Literaturwissenschaft»: la historia literaria como provocación a la teoría literaria (Jauss 1970).

Composición, construcción, estructura, código, símbolo, entre otros, son términos de uso común en arquitectura, literatura, matemáticas y música. La notación simbólica de la

${ }^{1}$ Coleridge, Samuel Taylor (1967): “On Poetry”, Times Literary Supplement 19: 1. 
que hacen uso matemáticas y música está muy próxima a la formulación química, a ciertos enunciados de la física teórica y al uso que de ellos hace la literatura, al menos desde los poemas futuristas de Marinetti, Russolo, Pratella, Cangiullo o De Nardis a partir de 1910.

En cuanto al concepto más actual de teoría que conviene ahora, por razones evidentes, es necesario ampliar los contornos que definen una teoría estrictamente literaria hacia los márgenes del ámbito científico, que siguen siendo igualmente válidos en esa disciplina. Cuando Einstein muere, en 1955, ya se habían combinado de forma coherente la teoría cuántica y la teoría de la relatividad, la gran conquista de Freeman Dyson y Richard Feyman. A partir de aquí, Lisa Randall, física teórica de la Universidad de Harvard, define por primera vez la teoría como marco físico encarnado en un conjunto de conjeturas básicas sobre el mundo, que proporciona un número específico de ecuaciones y predicciones confirmadas por su coincidencia con los resultados de los experimentos llevados a cabo para enunciarla (Randall, 2006). Los experimentos llevados a cabo en el contexto del arte sonorotécnico los hemos recogido hace años en nuestro estudio «Razones para un discurso progresista de la ciencia» ${ }^{2}$; las conjeturas de rango conceptual son dos definiciones que trascienden el término «música» en sentido convencional: «música es la organización de lo audioperceptible» y «música es la ciencia de mover los sonidos». Las ecuaciones la reduciremos a dos, la segunda de las cuales mantiene en principio una incógnita: «Texto es a estructura como arquitectura es a composición». «Matemática es a código como notación es $\mathrm{a}(\mathrm{x}){ }$

Recordemos que el primer texto de los Exercises de style de Queneau lleva por título «Notations» y que su idea de los Cent mil milliards de poèmes surge después del concierto en la sala Pleyel de París.

\section{CIENCIA DE SONIDOS Y PALABRAS}

La diferencia entre estética y ciencia del arte — Aesthetik y Kunstwissenschaft- que ha ocupado el debate en el ámbito germánico durante la primera década del siglo pasado, tiende a solapar, cuando no a confundir, ambos dominios (Calabrese, 1985). La ciencia, ámbito de origen de la música, ha sacudido los cimientos de la composición actual: teoría de fractales,

\footnotetext{
${ }^{2}$ Cureses, Marta: «Razones para un discurso progresista de la ciencia», en Mestres Quadreny, Josep M. (2011:
} 1-17). 
matemática combinatoria, series de Fibonacci, teoría de cuerdas, son solamente algunos de los procedimientos habituales entre los compositores de hoy. En el arte sonoro, el texto literario ha servido en muchas ocasiones para organizar el caos que genera una composición no regida por las reglas de la armonía tonal convencional. Cierto es que cuando el texto es no significante — pongamos la Ur Sonate de Kurt Schwitters- la estructura se complica, pero sigue brindando un marco que ayuda a sostener la construcción. De manera que, la presencia de elementos propios de la matemática —como en el Quadrivium: aritmética, geometría, astronomía y música - es algo natural que, es sabido, interesa muy pronto al OUvroir de LIttérature POtentielle (OuLiPo). Inventar estructuras o formas que permitan crear obras originales mediante la combinación de literatura y matemática, puesto que todos ellos son además matemáticos aficionados, admiradores del grupo Bourbaki o incluso, como afirma Jacques Roubaud, imitadores suyos. Cabe recordar asimismo que el proyecto fundacional de Bourbaki era reescribir las matemáticas en su conjunto y darles fundamentos a partir de una única fuente, la teoría de conjuntos, y de un método riguroso, el método axiomático (Roubaud, 1999).

Entre los miembros del OuLiPo destaca el poeta, ingeniero y músico Paul Braffort (París 1923-2018). Graduado en La Sorbonne en Ciencias (matemáticas), después de haber estudiado en el Institute Poincaré, y también en Filosofía, defendió en esta universidad una tesis doctoral sobre los fundamentos de las matemáticas. Su trabajo en el Commisariat de l'énergie atomique et aux énergies alternatives (CEA), en el EURATOM y en el Centre Européen de Technologie Spatiale (ESTEC) revirtió en sus constantes colaboraciones con el OUvroir. Su primera contribución al grupo, en el que ingresa el 13 de marzo de 1961, se presenta el 5 de junio de ese año y versa sobre las posibilidades de las máquinas electrónicas de cálculo. En el Commisariat había diseñado un nuevo sistema de clasificación de las materias con destino al Servicio de documentación; allí dirigió asimismo el Laboratoire de calcul analogique. Durante los años en el EURATOM y en el ESTEC, Braffort se encarga de la dirección de los centros de cálculo y las investigaciones en inteligencia artificial que por entonces estaban comenzando. Junto a Maurice Spighel y Christophe Tzara desarrolla una teoría física original: la electrodinámica estocástica. Tras la experiencia en el CEA se lanza de lleno a la aventura de la traducción automática. Cuando aún nadie en España hablaba de ordenadores, el EURATOM había adquirido un IBM 7090; Paul Braffort se las arregla entonces para visitar los centros de IBM en los Estados Unidos: «J'avais un équipe qui 
travallait notamment sur la traduction automatique, la démonstration automatique des théoremes, la simulation de jeu d'echecs» (Gavitto, 2014: 51).

En cuanto a su actividad literaria, Braffort había trabado amistad con Jean Paulhan en la Nouvelle Revue Française (NRF); pero los poemas que por entonces escribía no alcanzaban la calidad necesaria para ser publicados, como era su deseo, en la colección «Métamorphoses». Sin embargo, el contacto con Paulhan le lleva a conocer personalmente a Raymond Queneau, con quien comparte la pasión por las matemáticas y por los sistemas de clasificación de las ciencias. Como ejemplo de su pasión por estos sistemas, basta recordar su invención, a finales de los años setenta, de la «Bibliothèque Ordonée», un regalo para otro de sus colegas oulipianos, François Le Lionnais: sabiendo de su pasión por los números, diseñó una biblioteca cuyos volúmenes debían contener en los respectivos títulos cualquier tipo de número entero en expresión árabe, romana, cifras o formulaciones de otros tipos. Su ordenación ya venía dada.

Entre las empresas más complejas en las que se ve envuelto, tras su estancia en EURATOM y ESTEC, se encuentra el encargo de organizar los Centres de Traitement de l'Information en Bruselas, donde pone en marcha el estudio de algoritmos para el tratamiento de la lengua: «J'avais été recruté pour développer des algorithmes de traitement du langage natural en vue des applications à la documentation et à la traduction» (Gavitto, 2014: 53). En este proyecto, integrado por un equipo internacional procedente de Italia, Holanda y Francia trabajarían, junto a Braffort, Français Le Lionnais, Claude Berge y Max Euwe. Pero es a raíz de su nombramiento como profesor de la Universidad de París XI (Orsay 1971-1976) cuando orienta sus investigaciones hacia la lógica y la lingüística, lo que le lleva a dirigir la programación del Collège International de Philosophie, donde organiza los famosos seminarios «Science, art, littérature: noeuds et faisceaux culturels et leurs déploiments». Todos sus conocimientos estuvieron siempre a disposición del OuLiPo, especialmente de Jacques Roubaud, Marcel Benabou e Italo Calvino, junto a quienes fundó ALAMO (Atelier de Littérature Assistée para la Mathématique et les Ordinateurs), como veremos después.

La principal misión de los oulipianos, escritores y matemáticos, se había centrado en los problemas de la «création littéraire sous contrainte», con la que de alguna manera rendían homenaje a los «plagiaires par anticipation» que ya habían desarrollado este procedimiento mediante los lipogramas, palíndromos, etc. Pero la innovación oulipiana que suma los procesos matemáticos de composición a la escritura — y que ha dejado magníficos ejemplos en la obra de Perec o Queneau — va derivando hacia procedimientos de la 
combinatoria más rígida, lo que lleva a Paul Braffort y Jacques Roubaud a intensificar esta vía hasta desembocar en la informática, circunstancia que se separaba de los intereses iniciales del OuLiPo. De aquí surgirá la creación de ALAMO, nuevo grupo dedicado en exclusiva al binomio «Littérature/Informatique». A partir de la creación del Centro Georges Pompidou, el gobierno francés promoverá la creación mediante nuevas tecnologías: «Les compositeurs de musique, les graphistes, les plasticiens comme les écrivains devaient acceder à des outils d'analyse et de synthèse sophistiqués» (Gavitto, 2014: 55). Lo más singular y al tiempo interesante en esta personalidad tan poco común como la de Braffort es la actividad compositiva que desarrolla: cientos de canciones sobre textos de Raymond Queneau, Jacques Bens, Guillaume Apollinaire o Paul Éluard —entre ellas el Hymne à OuLiPo o Chez Borisademás de la música para la película La Joconde, con guión de Boris Vian y dirigida por Henri Gruel. La edición del disco Des atomes et des hommes (Pathé Marconi 1958) fue seguramente lo que le procuró una fama importante en el mundo de la música francesa.

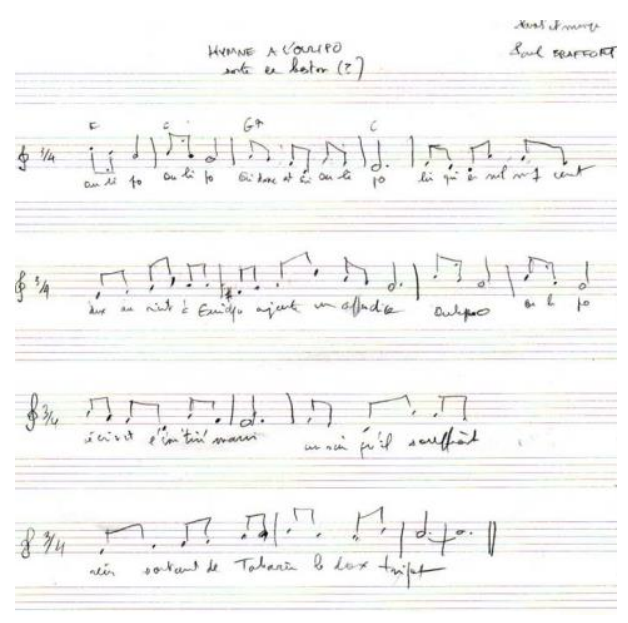

Figura 1. Braffort, Hymne à L'OuLiPo

En un sentido análogo se plantea el proyecto que Georges Perec crea junto a Marcel Benabou, bautizado como «Production Automatique de Littérature Française» o P.A.L.F, que se fija como objetivo «de trouver que n’importe quelle phrase disait la même chose que n’importe quelle autre» (Bellos 1994: 361). El procedimiento a seguir era siempre el mismo: reunían todos los diccionarios disponibles en el despacho de Perec, tomaban cada uno un volumen, bien del Littré o del Larousse, y se turnaban para leer en voz alta la definición de una palabra elegida al azar, mientras el que escuchaba transcribía cuidando de poner entre paréntesis la sintaxis del texto de origen para después construir sus propias frases: «C’était 
incontestablement le moment le plus difficile et le plus passionant...» ${ }^{3}$. A lo excepcional de este procedimiento hay que añadir que Perec utilizaba su máquina de escribir, no solamente como el medio eficaz de componer sus textos, sino como una auténtica caja de música. Es conocido el gusto dactilográfico de Perec, al punto que, como señala Jules Michelet, «avant d'écrire les livres, d'abord il les composait au sens pratique du terme» ${ }^{4}$. Le gustaban muchísimo las máquinas, en parte por el tacto de su teclado, pero especialmente por su sonido; así, durante los años sesenta escribía siempre con una Underwood, la misma que hizo de solista en la última obra radiofónica —börspiel_ que escribió por encargo de la Saarländdischer Rundfunk para esta radio alemana, el Konzertstück für Sprecher und Orchester, y cuya secuencia decimosexta se titula «Sonate für ältere Schreibmaschine» (Sonata para una vieja máquina de escribir). La obra, según las indicaciones, debe ser interpretada por una Underwood modelo Four Million.

Las creaciones de Perec y Braffort unen los dos extremos de las dos disciplinas, matemática y literatura, y en ambos casos añaden algo de música. Se entiende así la postura de Braffort respecto a la imposibilidad de separar vida científica y técnica de la artística y cultural, como ha explicado en su libro J\& I: les deux combinateurs et la totalité, donde parte de una estructura matemática de cálculo lambda de la que se deriva una estructura arborescente: “《J'ai donc pris l'arborescence comme organisateur du poème» (Gavitto, 2014: 55). Dice la poeta Clara Janés: «Amo las fórmulas / la inmovilidad de su perfección» ${ }^{5}$. Unos versos quizá inspirados en las palabras de Paul Valéry: las matemáticas, arte de la consecuencia, son una suerte de poesía de la repetición pura ${ }^{6}$. La poesía, como la música, tiene su propia sintaxis, y conviene recordar que, gracias a las matemáticas, las estrellas salen de la mitología para entrar en la astronomía.

Situemos ahora el concepto de repetición propio del minimalismo en el contexto literario. No somos originales en este sentido, puesto que Georges Perec ya eligió el símil con la música al emplear con soltura el término free jazz para este mismo propósito en su conocido texto «La chose» ${ }^{7}$. Perec decide denominar free jazz a lo que otros llaman

\footnotetext{
${ }^{3}$ Perec, Georges (1992): Cabiers Georges Perec III. Presbytères et Proletaires. Le dossier P.A.L.F., Paris, Éditions du Limon: 10.

${ }^{4}$ Michelet, Jules (1974): Le Peuple (1846), Paris, Flammarion: 26.

5 Janés, Clara (2010): V ariables ocultas. Con dos cartas de Antonio Gamoneda, Madrid, Vaso roto: 93.

${ }^{6}$ Discurso de Paul Valéry pronunciado en La Sorbonne el 30 de abril de1932 con motivo del centenario de la muerte de Goethe.

${ }^{7}$ Perec, Georges (1993): «La chose», en Le Magazine Littéraire n 316, décembre. El texto fue probablemente escrito en el año 1967.
} 
simplemente new thing, agrupando bajo esta denominación el conjunto de tentativas realizadas en el ámbito o dominio de la libre improvisación. El free jazz se inscribe así en el contexto de una problemática general de la estética contemporánea que interesa también a la literatura, de forma que el free jazz le permite hablar de escritura. Perec parte de los dos ejes de su sistema estético, contrainte y liberté, dispuestos en un sistema de coordenadas. Restricción y libertad como abscisas y ordenadas muestran las funciones indisociables de toda obra, pues la restricción no es más que la suspensión de la libertad, y la libertad no es más que la no restricción. En el caso de la música — que es uno de los ejemplos que Perec menciona expresamente en «La chose»— no puede hablarse (tanto si se trata del sistema tonal temperado como del sistema dodecafónico o serial) de un sistema más o menos libre, más o menos restrictivo, puesto que restricción y libertad constituyen precisamente el sistema. Podría parecer que el free jąz es entonces una paradoja, puesto que la improvisación reúne dos operaciones a la vez, creación y ejecución, pero lo hace empleando el mismo rigor que cualquiera de las formas clásicas conocidas. La explicación, en síntesis, se encuentra en los dos elementos propios de una pieza de este género: los elementos «negativos», encargados de romper la estructura tradicional que subyace en la obra, y los elementos «positivos», que son los que generan unidad y a partir de los cuales se desarrolla propiamente la nueva creación. Entre estos, Perec señala al menos dos que son conocidas figuras de la retórica: la repetición —el riff — y la cita.

La función de los riffs es casi la misma en el free que en el jazz en general: se trata de la figura fundamental de cohesión, la que une momentáneamente al grupo de intérpretes y en la que finalmente se resuelve la improvisación. Si bien es cierto que la función de la cita es más complicada, pues puede adoptar formas diversas, también es verdad que es el lugar común donde pueden inspirarse todos. La cita es, en un sentido más retórico que espacial, el lugar elemental para la improvisación ${ }^{8}$.

La conclusión de Hervé Le Tellier a partir de la frase de Raymond Queneau «Il n’y a de littérature que volontaire» se confirma en la sentencia de Claude Berge: «L’Oulipo, c'est l'anti-hasard» (Le Tellier, 2006: 8).

\footnotetext{
8 «La citation est donc le lieu (au sens plus réthorique que spatial) élémentaire de l’improvisation, le chemin ou, aun moins, le relais nécessaire de toute invention». Perec, Georges (1993): «La chose», Le Magazine Littéraire $\mathrm{n}^{\circ}$ 316, décembre.
} 


\section{AZAR CONTROLADO}

Son numerosos los estudios interesados en ahondar en las relaciones científicas que unen matemática y música, si bien desde una perspectiva actual el binomio se establece con mayor peso entre ciencia y arte sonoro. Los pitagóricos se anticiparon en la consideración de lo que llamaríamos «matemáticas puras», unas relaciones matemáticas por sí mismas, sin un fin inmediato. Pitágoras se siente atraído por la armonía musical puesto que esta consagra relaciones numéricas que pueden ser encontradas en cualquier otro lugar del universo. Su descubrimiento de las razones aritméticas entre intervalos armónicos le convence del vínculo sólido entre ambas disciplinas hasta el punto de establecer una conclusión: la música es el sonido de las matemáticas. Luego está, claro, el misterio que envuelve ese fenómeno extraño que se produce con el sonido simultáneo de notas: se combinan sin perder sus identidades; algo imposible cuando se trata de colores en la pintura, teniendo en cuenta, además, que frente a la instantaneidad del discurso visual, el recorrido sonoro se produce necesariamente en el tiempo.

La música y la ciencia en progreso es un trabajo de fondo que el compositor y científico catalán Mestres Quadreny (1929) ha ido escribiendo a lo largo de años dedicados a reflexionar sobre el pensamiento sonoro y científico, un laberinto en el que tiempo pasado y tiempo futuro apuntan siempre hacia el presente. Un discurso sincrónico de las innovaciones musicales y científicas que han determinado de manera conjunta su evolución a lo largo de siglos. Una suma de todos los ejercicios del pensamiento sonoro y científico desde los albores de la historia, cuando la música era ciencia antes que arte. Su formación en ciencias químicas le aproxima también al ámbito de la acústica y física cuántica. Resultado del estudio de estas disciplinas es el cambio de su concepto sobre las matemáticas. Abandona la idea de un complejo y sofisticado método de cálculo para adoptar la perspectiva de una visión abstracta: interpretar los fenómenos y crear los recursos necesarios para llevar a cabo valoraciones cuantitativas, de manera que el cálculo se admita simplemente como una consecuencia de todo ello.

La perspectiva desde la que Mestres plantea su teoría parte del conocimiento de los principales estudios en el ámbito internacional sobre el tema «arte sonoro y ciencia» en sentido amplio — Silbermann, Sir James Jeans Olson — o más precisamente sobre música y matemática — Harkleroad, Garland y Loy_, que representan momentos históricos de carácter sincrónico. En su planteamiento se contempla una Antigüedad en la que ya están 
presentes los sonidos sinusoidales, la frecuencia de onda, los decibelios, las variaciones de presión sonora y las células sensibles con las fibras del sistema nervioso, todo ello como materia prima de la que se nutre la música. Los valores estéticos de las matemáticas, a medio camino entre el arte y la ciencia, han sido objeto de estudio, entre otros, para Henri Poincaré, quien se refiere expresamente a la «belleza matemática» y la «elegancia geométrica». Ciertamente es preciso remontarse a los clásicos griegos para señalar, como Platón, que medida y proporción, esencia de la matemática griega, son sinónimo de belleza, mientras que para Aristóteles las formas que mejor expresan la belleza son el orden, la simetría y la precisión, conceptos todos ellos objeto de estudio de las matemáticas. La razón que dificulta la apreciación de la belleza en las matemáticas se encuentra, nos dice José Durán, en la carencia que nos impide percibir el valor estético de los razonamientos propios de esta disciplina: no somos capaces de discernir de manera automática la estructura tras la que se oculta esa belleza, algo todavía común también en el arte contemporáneo. Pero es sin duda la ordenación temporal que comparten música y matemáticas, esencial para ambas e irrelevante para la pintura, la que les confiere sentido: «Dicho en plata: un teorema, igual que una sinfonía, empieza, discurre y acaba, y el orden en que eso se produce es fundamental» (Durán, 2011: 39). Y lo que es más importante, ambos son lenguajes que discurren por fuera de la palabra.

Las teorías de los pitagóricos enlazan así en el proceder de Mestres Quadreny con el concepto de una métamusique preconizado por el arquitecto y compositor griego Iannis Xenakis —ingeniero colaborador de Le Corbusier en el Pabellón Philips y director del Centre d'Études Mathématiques et Automates Musicales de París- al hilo de su teoría del cálculo de probabilidades aplicado a la composición mediante una nueva propuesta que deriva en el principio estocástico como integrante de los procesos aleatorios. En el proyecto creativo de Mestres Quadreny, como en el de Raymond Queneau, se encuentran cent mille milliards de musiques cuya referencia inmediatamente anterior se encuentra en uno de los ejemplos más claros de condensación sonora: la obra de Anton Webern; en ella resulta evidente el relieve concedido a la cualidad de los sonidos individuales, en constante variación tímbrica y dinámica, así como la fluidez del discurso. Todo ello permite a Mestres diseñar un método personal cuya idea nuclear consiste en realizar variaciones sobre cada sonido, variaciones que interesan a la duración, la articulación, el timbre y la dinámica. En lo que respecta al timbre, en sintonía con la interpretación de Wittgenstein en Bemerkungen über die Farben, el lenguaje del color es un contexto lingüístico más. En el tratamiento que Mestres Quadreny aplica a 
sus construcciones, estos colores sonoros se presentan en grupos seleccionados entre el total cromático, tratados de manera diferente para cada proyecto. Sonidos grupales unidos por una relación temporal conjunta y regida por un sistema de proporciones. La obra inacabada, o más exactamente la obra sin fin, el proyecto de una creación que nunca se termina, es paradójicamente la meta que guía su impulso compositivo, y éste encuentra en las razones fronterizas del binomio música-ciencia una necesidad de apertura de esos mismos límites.

Desde muy temprano, Mestres se interesa por el efecto Tyndal y por el movimiento browniano, movimiento muy similar al de las moléculas de los gases estudiado en la mecánica estadística de Maxwell-Boltzmann. Esto le guiará después hasta los procedimientos compositivos sugeridos por la matemática estadística hasta alcanzar la creación de secuencias aleatorias. El poder creativo del azar está asimismo presente desde sus inicios como compositor. La obra del Premio Nobel 1965 Jacques Monod, Le hasard et la nécessité (1970), inspira una revisión de los conceptos «aleatorio»y «estocástico». Ambos designan fenómenos regidos por el azar, y contribuyen a esclarecer el común malentendido que hace equivaler el azar a lo fortuito; un esclarecimiento al que contribuye asimismo el también Premio Nobel 1977 Ilya Prigonine al establecer que la realidad no se sitúa ni en el puro azar ni en la necesidad, sino en una mezcla de ambos conceptos: la nietzscheana mano de hierro de la necesidad que sacude el cuerno del azar.

El azar por sí solo no sirve para crear nada; sin embargo, las acciones regidas por el azar en el contexto de determinadas contraintes pueden producir creaciones insólitas; las restricciones y límites establecidas por ellas sirven para canalizar estas acciones fruto del azar hacia objetivos concretos. Desde aquí, Mestres Quadreny se introduce en la especulación positivista que le proporciona el conocido método Montecarlo, un procedimiento que simula este tipo de procesos a partir de la estadística inductiva. El desarrollo de esta variante de la estadística se encuentra estrechamente relacionado con el cálculo de probabilidades. Conviene puntualizar que cuando aquí nos referimos a estadística inductiva lo hacemos para designar la rama orientada a interpretar mediante leyes de probabilidad las regularidades estadísticas que permiten extraer leyes de previsión de resultados. En cuanto al método Montecarlo, no es un procedimiento de composición en sí mismo, sino un instrumento del que se vale el compositor para establecer procesos automáticos de toma de decisiones.

En sus inicios como compositor, Mestres busca sus referentes fuera del ámbito musical — cabe recordar que Robert Gerhard se había exiliado en Cambridge el año 1937— y los encuentra en las líneas de acción de tres de sus mejores amigos: Joan Miró, Antoni 
Tàpies y Joan Brossa, este último uno de los mejores escritores de sextinas ${ }^{9} \mathrm{y}$ artífice de un itinerario que recorre la poesía clásica hasta una poesía visual de rasgos absolutamente personales. La línea desarrollada por Brossa parte de los textos de Arnaut Daniel, cuyas sextinas siguen en términos matemáticos una permutación de orden seis, como sucede en la que se considera primera sextina de la historia, «Lo ferm voler qu'el cor m'intra», cuya estructura es $123456=246531$. Tres textos de Brossa inspiran respectivamente los ballets compuestos por Mestres Quadreny titulados Roba i ossos, Petit diumenge y Vegetació submergida. Para él también escribe Cançons de bressol, el texto de su ópera El ganxo y de la Acció per a Joan Miró, además de participar juntos en la creación colectiva Cop de poma (1962) que integraba obras de Antoni Tàpies, Moisès Villélia, Mestres Quadreny, Joan Brossa y Joan Miró.

Su planteamiento, seguramente derivado de conceptos propios de las líneas de pensamiento de estos amigos, le lleva a concebir una música desprovista de sintaxis, lo que le permite diseñar cada obra musical como un objeto, planificando su desarrollo en bloques sonoros plasmados sobre papel milimetrado mediante grafías exactas. De manera que, cuando comienza a trabajar con procesos aleatorios, simplemente sustituye aquellos bloques sonoros por un nuevo concepto: los campos de probabilidad. El diseño de cada uno de estos campos consta de una descripción muy precisa de las leyes de probabilidad y de las limitaciones, que Mestres Quadreny denomina «constreñimientos» —contraintes-, destinados a controlar las alturas, densidades y otros parámetros sonoros. A partir de aquí se deja guiar por el azar aplicando las herramientas que le proporciona el ordenador —el proceso se inicia con los primeros ordenadores IBM que llegan a España-incrementando después la libertad expresiva mediante la ampliación del espectro de posibilidades hacia la dimensión lúdica de un azar controlado. Las teorías del azar y su control en la composición estaban aún sin explorar, pero, tratándose de referentes en el ámbito de la pintura, podemos ejemplificar su eficacia en la obra de Jackson Pollock, uno de los mejores ejemplos de aparente aleatoriedad.

Ese aspecto de compleja red de líneas continuas fruto del chorro de pintura vertida sobre el lienzo es en realidad una disposición orgánica ordenada. Los estudios de Richard P. Taylor sobre las obras del periodo 1947-1952 han revelado a través de un minucioso análisis de reconocimiento de pautas, que la disposición en los lienzos está acorde con fractales casi

\footnotetext{
${ }^{9}$ Brossa, Joan (1987): Viatge per la sextina 1976-1986. Barcelona, Quaderns Crema. Su participación en el grupo Dau al set es determinante para la evolución de su obra y también para la de Mestres Quadreny, que entabla gran amistad con Joan Ponç, Modest Cuixart, y muy estrecha con Tàpies.
} 
perfectos. Estas pautas empleadas son lo que se denomina «invariantes por escala», sin tamaño definido, y sirven para identificar la cantidad de información que se contiene en cada una de ellas. Este anticipo intuitivo de Pollock de lo que Benoît Mandelbrot desarrollaría después como una teoría matemática completa, revela una facilidad perceptiva fuera de lo común para identificar un aspecto estadístico a partir de la experiencia plástica. Lejos de ser un procedimiento analítico de signo anecdótico, el sistema ideado por Taylor ha sido empleado para resolver la autentificación de casi cincuenta lienzos atribuidos a Pollock, en función de esta presencia o ausencia de pautas fractales distintivas ${ }^{10}$. De forma que las pautas de Pollock no son casuales ni están regidas por el azar, aunque pudiera parecerlo: se ejerce un control sobre el azar.

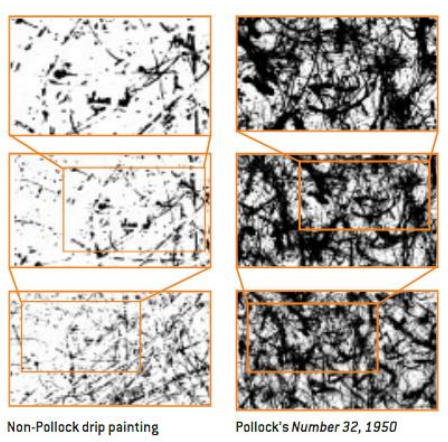

Figura 2. J. Pollock. Pautas de saturación

Una fórmula similar puede estudiarse en el empleo de pautas de manera sistemática en los frisos lineales de Oriente Medio. Las siete pautas lineales que pueden usarse en un friso geométrico responden a cuatro operaciones básicas: traslación, reflexión, rotación y reflexión con deslizamiento. Si se pretende reproducir diseños repetitivos, cada uno de estos cuatro movimientos básicos solo pueden combinarse entre sí de siete maneras diferentes: traslación, reflexión horizontal, reflexión horizontal con deslizamiento, reflexión vertical, rotación de $180^{\circ}$, reflexión horizontal/vertical y reflexión vertical/rotación (Barrow, 2005). Ahora bien, si no pretendemos conseguir diseños regulares con estructura reticular invariante, cualquiera de las pautas podría combinarse con las demás en un número infinito de permutaciones. El proceso inicial se produce de la misma manera en la teoría de los doce sonidos generada por Arnold Schoenberg; pensemos en las pautas compositivas del sistema dodecafónico empleado por los seguidores de la Escuela de Viena y que tiene las mismas

${ }^{10}$ Puede verse una valoración detallada en www.authenticationinart.org (último acceso 11/05/2019). 
cuatro posibilidades básicas: serie original, retrogradación, inversión e inversión retrogradada. El segundo proceso descrito para las pautas geométricas es equiparable a la aplicación de los principios de la combinatoria —-matemática combinatoria o estadística-a la composición por procedimientos estocásticos o método Montecarlo. Las posibilidades son aquí igualmente infinitas, como muestra, por ejemplo, la conocida obra de Steve Reich PianoPhase. Es muy conocida la experiencia llevada a cabo en el siglo XVIII por W. A. Mozart al componer un vals que presenta once variaciones posibles para catorce de sus dieciséis compases, añadiendo dos opciones para interpretar cada uno de los otros dos restantes. El cálculo de probabilidad nos ofrece aquí 2 x11 elevado a 14, o lo que es igual, una cantidad de valses suficientes para que un millón de parejas de baile puedan danzar durante unos dos millones de años. La idea de Cent mil milliards de poèmes de Raymond Queneau discurre en paralelo. La serie de Fibonacci empleada por Paul Braffort en «Hors-D’oeuvre», siguiendo los principios de Roubaud y el teorema de Zeckendorf, que permite representar el poema como la suma de elementos se la serie $-9=8+1,20=13+5+2$, etc. - es asimismo una manera de transferir la estructura matemática a una contrainte literaria de orden semántico: «Le contenu du poème de rang $n$ dépend du contenu des poèmes dont le rang forme la représentation “de ZECKENDORF” de n» (Bénabou, 2009: 158). Uno de los ejemplos más interesantes de Braffort son Mes Hypertrophes. Vingt-et-un moins un poèmes à programme, publicados en la Bibliothèque Oulipienne y el primero de los cuales está construido sobre las rimas: fi - bo - na - xi.

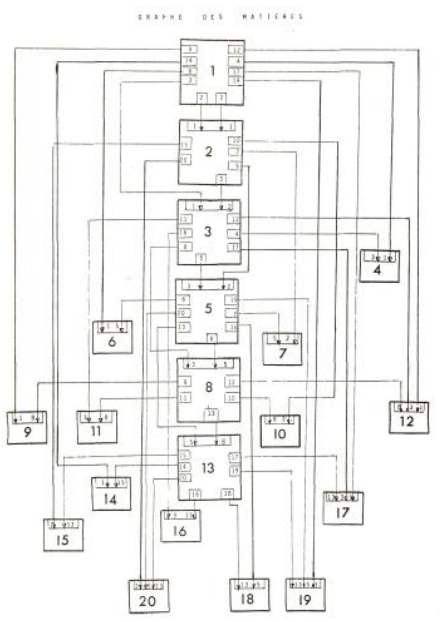

Figura 3. Braffort, Mes Hypertropes 
Suite bufa (1966) es el título de la primera aproximación de Mestres Quadreny a la composición mediante procesos de azar. En esta obra, cuyo proceso de construcción podría recordar a las instrucciones de Tristan Tzara «Pour faire un poème dadaïste» Mestres se inspira directamente en una litografía de Joan Miró publicada en Derrière le Miroir en la que se podían observar unas salpicaduras de pintura de apariencia aleatoria: lo que parecía fruto del azar era en realidad una perfecta imitación del mismo. La recreación del azar, permitiendo la intervención del mismo es lo que el compositor traslada a la partitura de forma singular: imitar un fenómeno de azar mediante procedimientos propios de azar. Y es este artificio de imitación del azar con intervención de la mano del artista el que desembocará en el empleo de procesos aleatorios.

La formación científica de Mestres Quadreny deja su impronta en concebir un proyecto monumental titulado L'Estro aleatorio (1973-1978), en alusión a L'Estro harmonico de Antonio Vivaldi, e integrado por seis conciertos para solista y orquesta: Concierto ${ }^{0} 1$ para violín y orquesta (1975), Concierto $n^{\circ} 2$ para guitarra y orquesta (1975), Concierto $n^{\circ} 3$ para máquina de escribir y orquesta (1975), Concierto $n^{0} 4$ para piano y orquesta (1976), Concierto $n^{0} 5$ para percusión y orquesta (1977) y Concierto n6 para grupo instrumental y orquesta (1978).

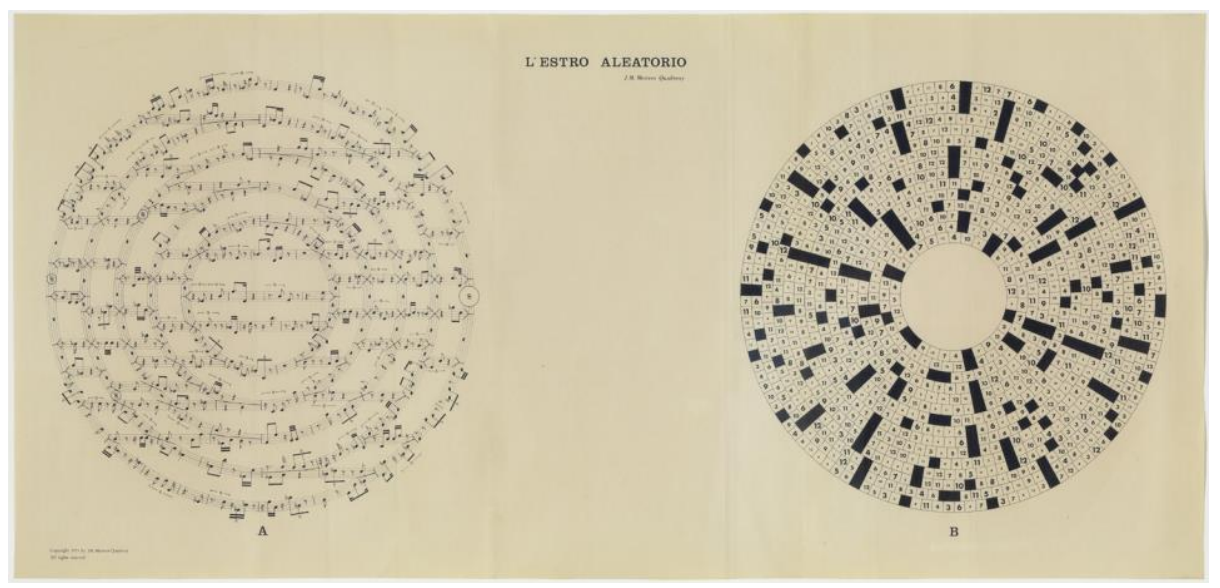

Figura 4. L'Estro Aleatorio (1973-1978)

Entre los procesos mironianos que Mestres ha importado a su propia obra se encuentra el proceso de metamorfosis empleado en sus tres primeras sinfonías, a la manera de los Intérieus hollandaises, así como la concepción objetual de la música y la introducción de procesos aleatorios como elemento de relación entre sonidos. 
El antecedente inmediato se encuentra en otra partitura suya, Aronada (1970), cuyo significado en sánscrito es «círculo de los sonidos audibles», concebida a partir de una tabla de números aleatorios sobre base 12 y otra tabla de intensidades. A partir de ellas construye una partitura generativa con todos los ingredientes para generar un flujo musical de duración indefinida e ilimitada y configuración abierta. Gráficamente está representada en forma circular e integrada por dieciséis círculos concéntricos, cada uno de los cuales, a modo de corona circular, está dividido en casillas que contienen un número que expresa el tiempo en segundos con asignación de dinámicas o intensidades, o bien son de color negro e indican una pausa y cambio de sonido. Las partituras de Aronada fueron realizadas en el estudio de arquitectura de Enric Tous y Josep Maria Fargas y editadas por Seesaw Music Corporation.

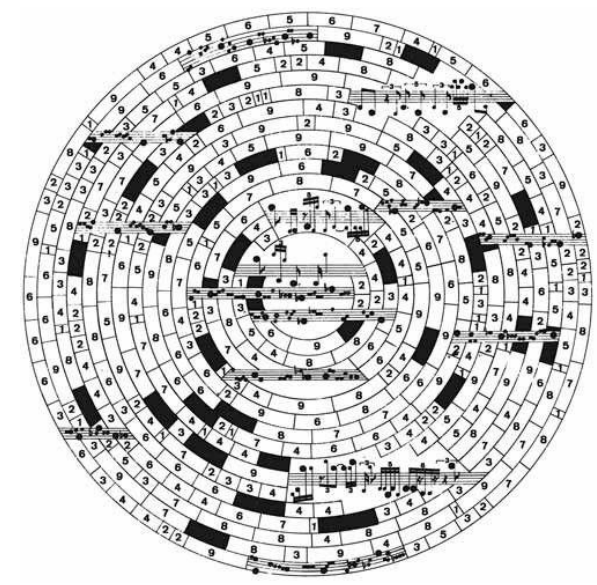

Figura 5. Aronada (1969)

En un futuro quizá primará la construcción sonora y textual autónoma frente al discurso lógico; y no tendrá relación con los cánones formales o estéticos que hemos conocido lo largo de la historia.

\section{MINIMALISMO MUSICAL Y POÉTICO}

La trayectoria de Michael Nyman puede explorarse a través de sus escritos y de sus primeras obras experimentales hasta la consolidación, en los años setenta del siglo pasado, de una madurez compositiva que contempla los movimientos artísticos ingleses de signo experimental y vira rápidamente hacia los logros de los ya clásicos minimalistas americanos de 
mediados de esa década, autores en realidad coetáneos. Sus intereses literarios determinan sus composiciones de principio a fin de su catálogo hasta el punto de convertirse en barreras que le impiden avanzar hasta no encontrar una resolución adecuada música-texto, como le sucede con su ópera inacabada sobre The Life \& Opinions of Tristram Shandy, y donde la pasión por los números le lleva a seleccionar el «Slawkenbergius’s Tale» del volumen cuarto, capítulo primero de la obra de Lawrence Sterne, para llevarlo al texto de su «Nose List Song» en la sección cuarta de su ópera inconclusa.

Nyman se ha referido muchas veces a su propia línea de creación como «música matemática». Los ejemplos en su obra son muchísimos, pero quizá uno de los más sobresalientes se encuentra en la partitura escrita para la película Drowning by Numbers del director Peter Greenaway, un film cuyo guion se escribe después de haber escuchado la música de Nyman con destino al mismo. La primera escena es significativa: una niña salta a la comba mientras va enumerando rítmicamente los nombres de las estrellas, contando desde el número uno hasta cien: «One Antares, Two Capella, Three Canopus, Four Arcturus, Five Agreetor, Six Anektor, Seven Duendlin, Eight Algerib (...) Ninety-nine Procyon, and a hundred Elektra». Ante la pregunta de una mujer de por qué detiene su enumeración en un ciento, si hay muchas más de cien estrellas, la niña responde: «A hundred is enough. Once you've counted to a hundred, all the other hundreds are the same» ${ }^{11}$. Es decir, a partir del primer ciento todos los demás son iguales, como sucede en el límite de las sucesiones, uno de los principios del cálculo infinitesimal. Las cien estrellas que cuenta la niña tienen una simetría con el número de secuencias en las que los números del uno al cien van apareciendo en escenas sucesivas de la película de Greenaway.

Esta práctica se repite en otras obras, por ejemplo, en las simetrías radiales en Decay Music (1976) para el cortometraje homónimo de Greenaway, donde emplea acordes del uno al cien. Este método que se basa en el principio de repeticiones de naturaleza diversa encaja con la estética minimalista y con la poética de la repetición. A propósito de la técnica de la variación y la repetición, Omar Calabrese insiste en su estudio L'etá neobarocca en que la repetición no significa en modo alguno un aspecto poco original. La composición barroca es un anticipo del manejo que el minimalismo hará de estructuras breves y perfectamente definidas, sobre las que después proyecta un proceso de desarrollo basado en estas dos técnicas.

${ }^{11}$ www.scripts.com/drowningbynumbers 7305 (último acceso 11/05/2019). 
Otra lectura del planteamiento de Nyman es contemplar la posibilidad de un hipotexto, como se plantea por ejemplo en su ópera Don Giovanni in Re, una más de sus numerosas creaciones junto a Greenaway durante los años setenta y mediados de los ochenta. En este caso el hipotexto sería el aria de Mozart que Nyman emplea como referencia, mientras que la composición del restante material actuaría como hipertexto. La idea de ampliar un hipotexto parece bastante adecuada al proceso que se sigue aquí, porque literalmente amplía los contenidos de la pieza precedente, pero establece con claridad una distinción entre el original y la copia, de manera que no ha lugar a confusiones. En el ejemplo de Drowning by Numbers antes mencionado, Nyman adopta un planteamiento metatextual, donde el metatexto (que aquí es el segundo movimiento de la Sinfonía concertante K. 364 de Mozart) asume la condición de «comentario» de otro texto. En la película de Greenaway, la música comienza a sonar justamente cuando la niña, en su juego de contar estrellas, llega a la número cincuenta y ocho, el mismo número de compás de la secuencia de cuatro compases de la Sinfonía concertante sobre la que se desarrolla la partitura de Nyman. Nuevamente se observa que la técnica de la repetición requiere un número reducido de elementos. Queneau concibe su proyecto de cien billones de poemas a partir de los sonetos que divide estrictamente por versos independientes; sin esta premisa sería imposible conseguir su propósito combinatorio infinito como forma de demostrar le mouvement en marchant. En la música sucede exactamente lo mismo: sería imposible pensar una obra como Piano-Phase de Reich a partir del desarrollo de una sinfonía completa, una sonata o un preludio.

Nyman y Greenaway coinciden en su línea de acción porque comparten la difícil simplicidad estructural que propone el minimalismo como medio de expresión, tanto desde un punto de vista técnico como estético. En cuanto a Greenaway, que tuvo en sus inicios una discreta actividad como dibujante y se aproximó a la arquitectura y las matemáticas, uno de sus procedimientos compositivos más habituales consiste en un vocabulario de imágenes y un catálogo de temas que va tachando de una lista a medida que los va usando, procedimiento inspirado directamente en Borges. Drowning by Numbers presenta una magnífica simetría en la mayoría de sus planos principales. Todos los números del uno al cien se muestran a lo largo de la narración, que está expuesta de modo fragmentario — acorde con el sentido minimalista de la música - con un carácter experimental y barroco. Su fijación con la estadística, la numerología y otros aspectos científicos son elementos omnipresentes en sus películas.

Cuando Pwyll ap Siôn, profesor en Bangor, Universidad de Gales, invitó a Michel Nyman a ofrecer una conferencia en el contexto del Oxford Contemporary Music Group, el 
tema elegido fue la explicación detallada de los procedimientos que había empleado para escribir la banda sonora de Drowning by Numbers a partir de la obra de Mozart ya citada. En el trasfondo del tema se encontraban las críticas vertidas sobre el procedimiento compositivo empleado por Nyman y al que el compositor, no solamente no es ajeno, sino que ha parodiado sobre el mismo; hay una escena en su tv-ópera Letters, Riddles and Writs (1991) — en homenaje a Mozart - en la que el compositor se somete a una especie de juicio sumarísimo al ser acusado de plagio musical por el mismísimo Franz J. Haydn. La acusación a la que responde el compositor se refiere a un pasaje de unos siete compases pertenecientes a la sección del desarrollo del primer movimiento del cuarteto en mi bemol mayor K. 428 de Mozart. Actúa como su abogado el musicólogo suizo Georg Nägeli, que sostiene su defensa citando un pasaje del manual de Carl Czerny School of Practical composition según el cual «one must compose according to chosen models, taking the same key, time, phrase structure, number of bars and even modulation, following them strictly» (Siôn, 2007: 1). Desde luego, las críticas a Nyman han sido reales, aunque no muy contundentes. En numerosas ocasiones lo han hecho Keith Potter ${ }^{12}$ o Meirion Bowen, quien se arriesga aún más al afirmar que si Nyman posee algún estatus como compositor — cuestión que duda - lo ha adquirido «by steadily annihilating all past composers from Purcell to Mozart» ${ }^{13}$. El propio Nyman ha respondido de manera firme: «Whenever I borrow... I always credit my sources» ${ }^{14}$, lo cual es cierto.

El estudio fonoestilístico de los poemas de Raymond Queneau realizado por Renée Baligand se detiene en analizar el empleo de las anáforas como recurrencias sonoras que aportan un efecto de compensación rítmica al conjunto. Su empleo se estudia desde los planos vertical y horizontal que sirven para realizar una clasificación de su uso. Podría establecerse así un paralelismo entre las anáforas y las citas reiterativas propias del minimalismo musical. Se repite una palabra (sonido, acorde) o estructura gramatical (o musical) en diferentes versos (o compases). En la obra de Queneau las anáforas se usan expresamente para añadir sonoridad al poema, «par leurs récurrences sonores specifiques» (Baligand, 1972: 38), generando en ocasiones una suerte de equilibrio rítmico compensatorio. Además, lo que es más relevante, su disposición en el verso es tanto vertical como horizontal, que en la partitura nos remite al sentido armónico y melódico respectivamente. De los

12 Potter, Keith (1991): «New Music», en Musical times, 131: 212-213.

13 Bowen, Meirion (1995): «Synthetic material», en The Guardian, 10 april: 9 (citado por Siôn, 2007).

14 Webster, Jonathan (1995): «The Composer’s Contract», en Gramophone Magazine, april: 14-17. 
muchos tipos de anáfora utilizados por Queneau, uno de los más interesantes es la anáfora de sintagma, empleada con frecuencia en la Petite cosmogonie portative: aquí, un sintagma, por ejemplo verbal, seguido de una proposición relativa, prolonga su estructura anafórica hasta prácticamente invadir todo el verso. Nace así un procedimiento muy particular de Queneau: en lugar de la anáfora misma puesta de relieve, lo que se realza o subraya es la parte final del verso, una parte no anafórica:

«Une bête elle est là gigotant dans le bleu une bête elle est là elle saute et volette une bête est là molle et poussant ces yeux une bête est là fille de ces ancêtres (...)».

Como en la composición minimalista, las estructuras repetitivas van añadiendo un elemento nuevo a cada aparición. También en el empleo de la anáfora sintáctica se forma una especie de ritmo sincopado que se repite, por ejemplo, en Si tu t'imagines, en seis cuartetos, lo mismo que en las anáforas de palabras, en las que la insistencia sonora crea un clima musical salmódico, y la brevedad que impone la palabra realza su fuerza sonora. En esta clase de anáfora Queneau hace uso de todos los tipos posibles: sustantivos, verbos, adjetivos, pronombres, preposiciones, adverbios e incluso cifras o sucesiones de números. En sus «Concordances Baudelairiennes» cada verso viene determinado por una combinación numérica:

004508 sur Paris dormant ruisselait 089007 le vieux Paris n'est plus la forme d'une ville. 089029 Paris change! Mais rien dans ma mélancolie 091026 traversant de Paris le fourmillant tableau. 103027 Et le sombre Paris en se frottant les yeux (Courir les rues, 41)

Los juegos y combinaciones o formulaciones del cálculo infinitesimal, como sucedía en Drowning by Numbers de Greenaway -1 Antares, 2 Capella, 3 Canopus... - se convierten en una constante vital para Queneau, que se deja llevar por esta pasión incluso cuando envía una postal: 


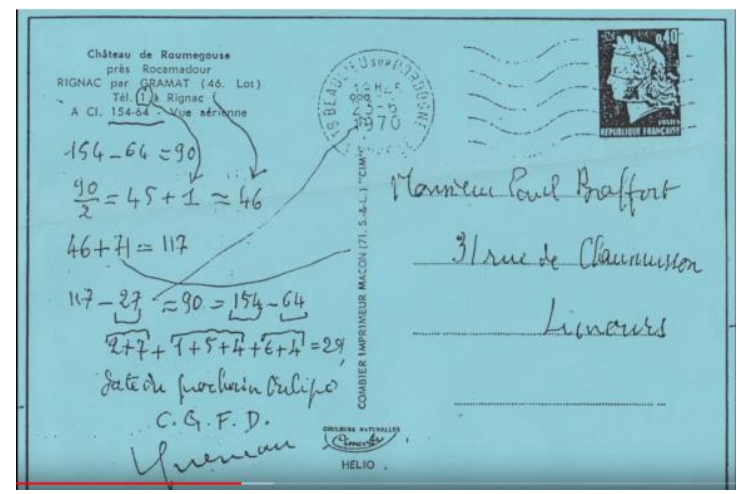

Figura 6. Tarjeta postal de Raymond Queneau a Paul Braffort 23/06/1970

\section{ARQUITECTURA COMO TEXTO: EISENMAN Y DERRIDA}

El capítulo quinto de Le modulor comienza así: «Mi trabajo se nutre desde hace más de treinta años de savia matemática, puesto que en mí la música está siempre presente» (Le Corbusier, 1953: 121). Ratifican esta sentencia las palabras de Steiner en la introducción a Lecturas, obsesiones y otros ensayos, donde sostiene que cualquier pensamiento sobre las formas del significado tiene que vérselas con la música. La cuestión de si puede o no establecerse un criterio firme sobre los significados de la música o « si se pueden o no aplicar criterios lingüísticos a un medio en el que contenido y forma constituyen una unidad, es asunto de enorme interés» (Steiner 1990: 25).

Podemos coincidir con Steiner en que la formulación de la geometría analítica, la teoría de las funciones algebraicas, el cálculo de Newton hasta llegar a «las grandes arquitecturas de forma y significado concebidas por Gauss, Cauchy, Abel, Cantor y Weierstrass se alejan del lenguaje a un ritmo cada vez más acelerado» (Steiner 1990: 380). Dicho de otro modo, su lenguaje expresivo adopta y elabora un vocabulario propio al mismo nivel que el discurso verbal. Esto mismo sucede en la arquitectura — también en las artes plásticas_-, especialmente a partir de la crisis de identidad experimentada por el movimiento moderno en los años sesenta del siglo pasado. Entre la multiplicidad de posibilidades alternativas el minimalismo y el pop-art empujaban en direcciones opuestas. Para los minimalistas representaba regresar a un grado cero de la escritura «a partir del cual construir, cautelosamente, unos mínimos significados» (Solà-Morales 2003: 33) ${ }^{15}$.

${ }^{15}$ El capítulo dedicado a "Mies van der Rohe y el minimalismo" fue originalmente publicado en Mertins, Detlef (ed.) (1994), The Presence of Mies, New York, Princeton Architectural Press, 149-155. 
Nos hemos referido antes a la trascendencia de las aportaciones de Einstein, cuya teoría de la relatividad dio lugar a un nuevo concepto de espacio asociado a la noción de tiempo, una relación, la de espacio-tiempo, que permeabiliza en la arquitectura: las renovaciones constantes en esta disciplina proceden singularmente de los cambios en las nociones espaciales. Si la arquitectura, como dice Louis Kahn, es la creación meditada de espacios, la música será la creación meditada de tiempos en sonidos y silencios. Oír un sonido es ver su espacio: «Los espacios en arquitectura me hacen desear componer una especie de música, figurándome la fusión de las disciplinas y de sus órdenes» ${ }^{16}$. Por otra parte, la propuesta formulada por Mijail Batjin que apunta hacia una estética literaria de las formas arquitectónicas resulta «un objetivo necesario para dotar de sentido e interés a la especulación crítica y teoría sobre la literatura» (García Berrio, 1989: 10). Un paso más allá nos lleva a la equiparación de las formas composicionales de la sintaxis con las formas arquitectónicas de la construcción (Batjin, 1978).

Si profundizásemos sobre la relación espacio tiempo en los ejemplos de arquitectura efímera, como el Pabellón Philips alzado por Le Corbusier y Xenakis, veríamos que muchos de los estudios realizados sobre este tipo de construcciones toman como referencia la noción de estructura sonora y especialmente el de tema con variaciones (Capanna, 2000), y lo mismo podemos decir de construcciones poéticas como las 35 Variations sur un thème de Marcel Proust (1974) de Georges Perec que, como es sabido, parten de un material prexistente, la primera frase de $A$ la recherche du temps perdu: «Longtemps je me suis couché de bonne heure». Pese a tener cierto parentesco con los Exercises de style, en la obra de Queneau el lector advierte las transformaciones de la breve historia en cada una de sus variantes, mientras que la lectura de Perec nos lleva hacia la variación puramente formal; de manera que la obra de Queneau transcurre para el lector como cuando se escuchan unas variaciones musicales en su forma clásica, mientras que la propuesta de Perec está más en consonancia con el nuevo concepto de forma en la música posterior a las vanguardias de principios del siglo pasado.

Respecto al trabajo de Edgar Varèse para el Poème Éléctronique del Pabellón Philips, es evidente que anticipa el concepto de instalación sonora, adaptándose al diseño reticular de las cubiertas paraboloides del edificio encargado por la casa holandesa OSRAM. El discurso poético es aquí una cuestión de base que suscita debates en el ámbito de la

${ }^{16}$ Kahn, Louis: «L'Architecture d'Aujourd'hui», conferencia pronunciada el 14 de noviembre de 1967 en New England Conservatory en el marco del simposio dedicado a la nueva fundación de este centro. 
arquitectura contemporánea: el «Poème de l’angle droit» (1955) de Le Corbusier encuentra respuesta en «Poema da curva» del arquitecto brasileño Oscar Niemeyer.

El arquitecto americano y profesor de la Universidad de Yale Peter Eisenman (New Jersey, 1932) ha dedicado buena parte de su carrera a decantar la forma arquitectónica hacia una ciencia teórica que se aparta del concepto semántico de sus elementos. Sus preocupaciones por la cuestión formal le han llevado al intento de conciliación de algunos de los aspectos más controvertidos de su propia obra, tratando de integrarlos en una suerte de teoría exhaustiva de la forma. Después de su estancia en Cambridge, de regreso a Nueva York, se aproxima al minimalismo, al arte conceptual y a la teoría estructuralista del arte. Sin embargo, lo más interesante de la obra de Eisenman para nuestro estudio es el autoanálisis que realiza sobre su planteamiento conceptual con sentido eminentemente crítico poniendo de manifiesto la pluralidad de elementos externos a su disciplina que manifiestan un discurso similar al que él lleva a término en la arquitectura, bien en el ámbito del arte, de la filosofía, de la literatura y, especialmente, de la lingüística, dominios en los que «los problemas formales se presentan bajo una óptica crítica y ofrecen un repertorio de técnicas extradisciplinares y categorías epistemológicas susceptibles de ser incorporadas en el discurso arquitectónico» ${ }^{17}$. Mediante la búsqueda de analogías entre el proceso constructivo de la arquitectura y la sintaxis derivada de la teoría postestructuralista propuesta por Noam Chomsky, Eisenman se decide a configurar una forma-texto como manera de integrar los procedimientos sintácticos y las unidades mínimas implicadas en el proceso. El ámbito del significado queda al margen del proceso; los componentes, sus signos, no se integran en un discurso coordinado a la manera de una lengua; «la analogía entre arquitectura y lingüística enseña que los elementos están subordinados al sistema» (Linazasoro, 2017: 18). El planteamiento que Eisenman realiza de la relación analógica arquitectura-lenguaje le lleva a establecer que la estructura de un entrono lingüístico tiene características similares a las de un entorno físico como la arquitectura; lenguaje y arquitectura pueden entenderse a su juicio dentro de las tres categorías semióticas, con un peso de la dimensión sintáctica mucho mayor en la arquitectura que en la lengua escrita. En la teoría de Peter Eisenman, el paso de la composición tonal a la dodecafónica o el paso de la prosa narrativa a la no narrativa responden a un mismo hecho: el cambio en la concepción de la relación entre el ser humano y su mundo objetual, una relación en la que el creador ya no se interpone entre el objeto y su significado. Esta

17 Pla Català, Anna: «Una genealogía de sistemas», (Eisenman, 2017: 14). 
conclusión le lleva a considerar la arquitectura como el conjunto de normas extraídas del universo de las formas y de «la acción de fijar unas condiciones geométricas, de uso y de significado que dan lugar a una nueva clase de objetos. En este sentido, es un sistema de signos» (Eisenman 2017: 103).

En su planteamiento de madurez hay un cambio significativo, especialmente a partir del descubrimiento de Jacques Derrida, con quien le une gran amistad, mutua admiración e intercambio de pensamientos y reflexiones; de todo ello da cuenta la correspondencia entre ambos y la edición de sus Choral Works (Derrida, 1996). La primera consecuencia —o quizá ya estaba antes en su cabeza- es la consideración de la arquitectura como texto. Cuando Eisenman se refiere a la poética potencial del texto arquitectónico sugiere la idea de una arquitectura entendida como escritura, totalmente opuesta a la idea de arquitectura como imagen. Su lectura debe hacerse a través del sistema de signos que denomina «huellas», un concepto que toma prestado directamente de Derrida en el sentido de la imposibilidad del objeto como representante de una realidad: «La arquitectura se convierte en un texto más que en un objeto cuando se concibe y se presenta como un sistema de diferencias, y no como una imagen o una presencia aislada» (Eisenman 2017: 131). La huella no debe leerse literalmente; simplemente señala la idea de lectura. Una exposición más concisa de su teoría la desarrolla en las reflexiones dedicadas a la arquitectura como segundo lenguaje —a la manera que concebimos una segunda lengua en nuestro aprendizaje- y a lo que denomina «textos intermedios». Allí plantea la noción de arquitectura como texto, fundamentando su idea de texto desde la multivalencia esencial del término: «El texto jamás admite un único significado y muestra cómo todo significa más de una cosa» (Eisenman 2017: 174). A esta idea suma la de «diagrama», una relación de fuerzas cuyo estado es una combinación de aleatorio y de dependiente. Falta añadir la incorporación del proceso de deconstrucción que Eisenman introduce en la arquitectura directamente importado desde Derrida, y que aplica a una interpretación del texto-objeto nada fácil de descifrar, salvo a partir de las construcciones físicas, de las obras que Eisenman ha levantado desde estos presupuestos. Entre ellas la más representativa a nuestro juicio es el Memorial to the Murdered Jews of Europe (Berlín 1998-2005), un conjunto espartano y poderoso, un austero y rotundo staccato. La visión minimalista de Eisenman procede aquí de la experiencia, no de la idea. 


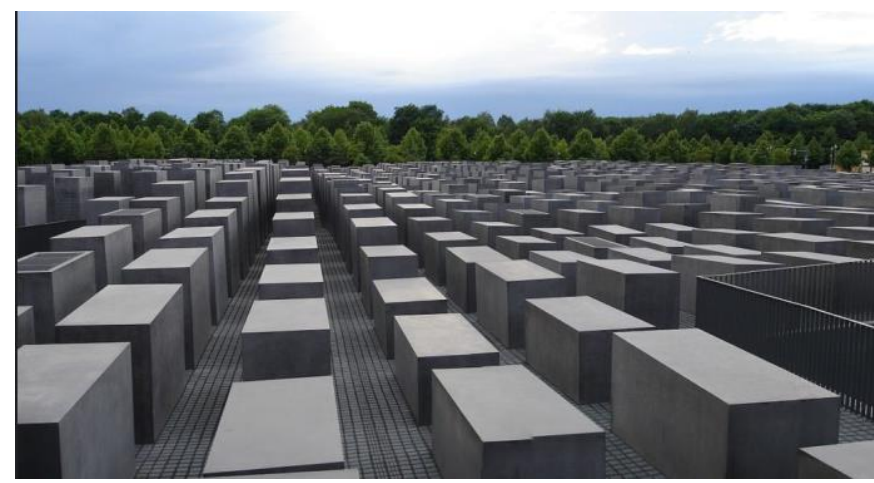

Figura 7. Eisenman Memorial to the Murdered Jews of Europe

La aproximación de Eisenman al autor de Cartesian Linguistics y la amplia influencia del método chomskiano y el sistema de diagramas permiten trasladar a los elementos arquitectónicos estructuras del lenguaje que dotan al resultado final de una sintaxis equilibrada, correcta, sin olvidar que el lenguaje, antes que forma, es sonido. El factor temporal se resuelve siguiendo las pautas que Derrida describe en sus escritos sobre la intervención de la arquitectura (Derrida, 2015). Apoyándose en ellos, y en L'écriture et la différence (Derrida 1967), Paul de Man señala hasta qué punto la deconstrucción aniquila cualquier estructura percibida como unitaria y monádica, si bien permite detectar fragmentaciones de otro modo imperceptibles ${ }^{18}$. En el contexto literario, mientras que el tiempo rige el sentido lineal de una escritura lógica, «el dominio multiforme de la escritura poética hace estallar la sucesividad lineal del tiempo» (García Berrio, 1989: 276) en lo que se revela como infinita superposición polisémica. Es decir, que es en el dominio del discurso poético donde el concepto espacial del código que conforma la estructura textualarquitectónica cobra verdadero sentido. La arquitectura como texto se extiende así a una arquitectura como texto poético. Y aquí el significado, como en la música, es claramente secundario. $\mathrm{O}$ incluso nulo.

\section{TRASCENDER EL AZAR}

Hemos tratado de resolver la tensión interdisciplinar entre conceptos y procedimientos que ayudan a generar una teoría del azar controlado a través de la praxis

\footnotetext{
${ }^{18}$ Una explicación completa de su teoría puede leerse en De Man, Paul (1979): Allegories of Reading: Figural
} Language in Rousseau, Nietzsche, Rilke and Proust, New Haven, Yale University Press. 
multidisciplinar. La composición minimalista, a través de la literatura y la ciencia, ha tomado aquí como referencia textos de Queneau, de Perec, de Braffort y música de Nyman y Mestres Quadreny inspirados en la matemática combinatoria y procedimientos aleatorios como parte de sus argumentos constructivos. Respecto al azar, Stéphane Mallarmé ofrece una sólida defensa en su conocido poema «Un coup de dés», que desde su segundo verso ya anuncia que el azar no se abolirá jamás. Poéticamente describe también Ian Hacking (1990), en su teoría de la domesticación del azar, lo que adopta como consuelo ante la imposibilidad de esa abolición, imagen del naufragio matemático: si no puedes dominarlo, trasciéndelo.

La teoría del azar controlado no ofrece un conjunto de soluciones, sino la expectativa de pensamiento futuro, un proyecto de pensamiento en marcha. Es natural que, como afirma Todorov (1969) parafraseando a Goethe, no pensemos de igual manera sobre un tema antes y después de haber escrito sobre él: negar este principio es reducir el texto a ese razonamiento que previamente hemos construido antes de emprender su desarrollo a partir de preguntas y respuestas que van surgiendo a cada paso. Más aún si admitimos, como él admite en Les genres du discours (1978), que cada tipo de discurso calificado como literario tiene padres no literarios más cercanos a él que cualquier otro tipo de discurso literario. Esta circunstancia parece evidente en todos los ejemplos analizados en estas páginas. La interpretación que se ha expuesto hasta aquí, funciona como lectura interpuesta, tomando la expresión de Barthes (1967), con el fin de esclarecer las posibilidades que ofrece una teoría del azar controlado a través de la literatura, la música, y la arquitectura, sin olvidar que todas y cada una de estas disciplinas no son sino visiones parciales del mundo.

Muchas de las poéticas que han utilizado el azar y los procedimientos aleatorios en sus producciones lo hacen no solamente como un modo de provocar movimientos en las estructuras estables, sino como demostración de la imposibilidad de conocer la totalidad de estructuras posibles. Y aquí, como en las arquitecturas de piedra, dice Louis Kahn, cada piedra resulta más importante que la cantera.

\section{$[\ldots \ldots . .$.}

—Así que tienen diez mil piedras en un prado — dijo Nashe—y no saben qué hacer con ellas.

- Ya no es así —respondió Flower-. Sabemos exactamente lo que vamos a hacer con ellas. ¿Verdad Willie?

—Desde luego —afirmó Stone, sonriendo repentinamente con alegría- Vamos a construir un muro. 
Ue Actio nova: REVISTA DE TEORÍA DE LA LITERATURA Y LITERATURA COMPARADA, 3 (2019): 407-438

—Un monumento, para ser más precisos — dijo Flower-. Un monumento en forma de muro. En mi opinión, no hay nada más misterioso ni bello que un muro. Ya lo estoy viendo: levantándose como una enorme barrera contra el tiempo. Será un monumento conmemorativo de sí mismo, caballeros, una sinfonía de piedras resucitadas, que cada día cantará una endecha por el pasado que llevamos en nuestro interior ${ }^{19}$.

19 Auster, Paul (1990): La música del az̧ar, Barcelona, Seix Barral: 45 


\section{BIBLIOGRAFÍA}

Baligand, Renée (1972): Les poèmes de Raymond Queneau. Étude Phonostylistique, Ottawa, Didier. Barrow, John D. (2005): The Artful Universe Expanded, Oxford, Oxford University Press.

Barthes, Roland (1967): Système de la mode, Paris, Seuil.

Barthes, Roland (1984): Le bruissement de la langue, Paris, Éditions du Seuil.

Batjin, Mijail (1978): Esthètique et théorie du Roman, Paris, Gallimard.

Bellos, David (1994): Georges Perec. Une vie dans les mots, versión francesa del original inglés por Françoise Cartano y David Bellos, Paris, Éditions du Seuil.

Bénabou, Marcel; Paul Fournel (ed.) (2009): Anthologie de l'OuLiPo, Paris, Gallimard.

Calabrese, Omar (1985): Il linguaggio dell'arte, Milano, Bompiani.

Capanna, Alessandra (2000): Le Corbusier. Padiglione Philips, Bruxelles, Torino, Capolavori Testo \& Immagine.

Culler, Jonathan (1997): Literary Theory. A Very Short Introduction, Oxford, Oxford University Press.

Derrida, Jacques (1967): L'écriture et la difference, Paris, Seuil.

Derrida, Jacques (2015): Les arts de l'espace. Écrits et interventions sur l'architecture, Paris, Éditions de la différence.

Derrida, Jacques; Peter Eisenman (1996): Choral Works, New York, The Monacelli Press.

Durán, Antonio J. (2011): La poesía de los números. El rol de la belleza en las matemáticas, Barcelona, RBA.

Eagleton, Terry (1983): Literary Theory, Oxford, Basil Blackwell Publishers Limited.

García Berrio, Antonio (1989): Teoría de la literatura (La construcción del significado poético), Madrid, Cátedra.

Gavitto, Jean-Louis; Valérie Schafer (2014): "Paul Braffort, un pied dans la littérature, un pied dans la science", en Bulletin de la société informatique de France, 3: 49-57.

Eisenman, Peter (2017): 11+L. Una antología de ensayos, traducción al español de Maurici Pla y Moisés Puente, Barcelona, Puente editores.

Hacking, Ian (1990): The Taming of Chance, Cambridge (UK), Cambridge University Press.

Le Corbusier (1953): El Modulor. Ensayo sobre una medida armónica a la escala bumana aplicable universalmente a la arquitectura y a la mecánica, traducción al español de Rosario Vera Buenos Aires, Editorial Poseidón.

Le Tellier, Hervé (2006): Esthètique de L’Oulipo, Bordeaux, Le Castor Astral. 
Linazasoro, José Ignacio (2017): Textos críticos, Madrid, Ediciones asimétricas, Departamento de Proyectos, ETSA, Universidad Politécnica de Madrid.

Mestres Quadreny, Josep M. (2011): La música y la ciencia en progreso, Tarragona, Arola Editors.

Jauss, Hans Robert (1970): Literaturgeschichte als Provokation, Frankfurt, Suhrkamp Verlag.

Queneau, Raymond (1960): Cent mille milliards de poèmes, Paris, Gallimard.

Randall, Linda (2006): Intelligent Thought: Science versus the Intelligent Design Movement, New York, John Brockman, Vintage.

Roubaud, Jacques (1999): Poesía, etcétera: puesta a punto, traducción al español de José Luis del Castillo Jiménez, Madrid, Hiperión.

Sîn, Pwyll ap (2007): The Music of Michael Nyman. Texts, Contexts and Intertexts, Hants (England), Ashgate.

Solà-Morales, Ignasi (2003): Diferencias. Topografía de la arquitectura contemporánea, Barcelona, Gustavo Gili.

Steiner, Georges (1990): Lecturas, obsesiones y otros ensayos, Madrid, Alianza Tres.

Todorov, Tzvetan (1969): Grammaire du Décaméron, The Hague, Mouton \& Co. N. V. Publishers. 


\section{SOBRE EL AUTOR}

\section{Marta Cureses}

Licenciada en Filología Anglogermánica (Universidad de Salamanca), Doctora en Geografía e Historia (Universidad de Oviedo) y profesora titular del Departamento de Historia del Arte de esta Universidad. Miembro del GIR Ilicia (Inscripciones literarias de la ciencia, Universidad de Salamanca), del TEI (Texto e Imagen, Universidad de Oviedo) y de consejos y comités científicos. Colaboradora en trabajos editoriales, congresos, exposiciones y conferencias en la Fundación Juan March, Centre Santa Mònica, Instituto Cervantes, Fundación Joan Miró, La Pedrera-Fundación La Caixa, Espai d'Art Contemporani de Castellón, Fundación Brossa, La Casa Encendida y Museo de Navarra. Ha publicado libros y artículos en medios nacionales e internacionales. Como Directora del Área de Cultura de la UO ha dirigido ciclos de conferencias de Aula Magna, actos Fundación Príncipe de Asturias, exposiciones, edición de trabajos literarios y científicos, y textos de catálogos. Coordinadora general del Doctorado Interuniversitario con Mención de Calidad (MDC2005-00216) en la UB, UGR, UO y USC. Como Subdirectora General del Ministerio de Cultura ha desarrollado trabajos de gestión en los principales organismos de las CCAA. Sus libros más recientes: Joan Miró-Mestres Quadreny. Suite Miroir (Fundació Joan Miró de Barcelona- UPF 2017) y Nuevos caminos del arte y de la ciencia. Premio Internacional Jaén (2019).

Contact information: Universidad de Oviedo. Campus de Humanidades. Edificio Departamental. 33011 Oviedo. Tel. 34+985104486. Email: cureses@uniovi.es. www.ilicia.es. 